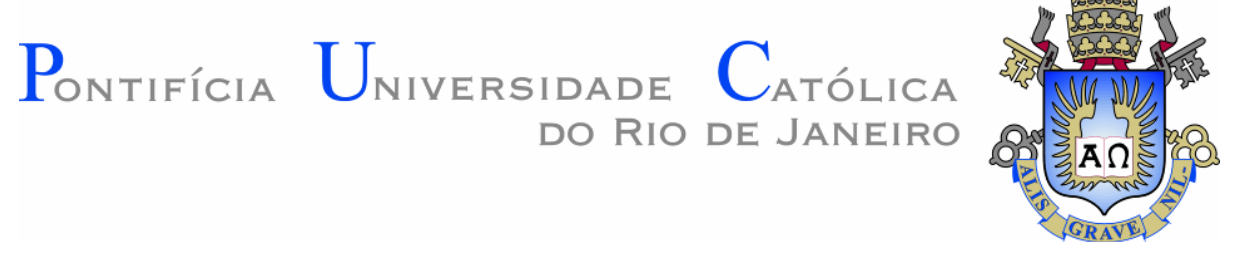

Diego Aguiar Fonseca

Previsibilidade na dinâmica da superfície de volatilidade implícita em opções de compra de ações brasileiras

\begin{abstract}
Dissertação de Mestrado (Opção profissional)
Dissertação apresentada como requisito parcial para obtenção do título de Mestre pelo Programa de PósGraduação em Administração da PUC-Rio.

Orientador: Prof. Antonio Carlos Figueiredo Pinto
\end{abstract}


Diego Aguiar Fonseca

\title{
Previsibilidade na dinâmica da superfície de volatilidade implícita em opções de compra de ações brasileiras
}

\begin{abstract}
Dissertação apresentada como requisito parcial para obtenção do título de Mestre pelo Programa de PósGraduação em Administração da PUC-Rio. Aprovada pela Comissão Examinadora abaixo assinada.
\end{abstract}

Prof. Antonio Carlos Figueiredo Pinto

Orientador

Departamento de Administração - PUC-Rio

Prof. Antonio Carlos Figueiredo Pinto Departamento de Administração - PUC-Rio

Prof. Marcelo Cabus Klotzle Departamento de Administração - PUC-Rio

Roberto Marcos da Silva Montezano IBMEC/RJ

Profa. Mônica Herz

Coordenador(a) Setorial do Centro de Ciências Sociais - PUC-Rio 
Todos os direitos reservados. É proibida a reprodução total ou parcial do trabalho sem autorização da universidade, do autor e do orientador.

\section{Diego Aguiar Fonseca}

Graduou-se em Administração de Empresas pela Universidade Federal do Rio de Janeiro em 2008. Responsável pela Área de Controle de Investimentos na empresa Icatu Seguros S.A.

Ficha Catalográfica

Fonseca, Diego Aguiar

Previsibilidade na dinâmica da superfície de volatilidade implícita em opções de compra de ações brasileiras / Diego Aguiar Fonseca ; orientador: Antonio Carlos Figueiredo Pinto. - 2013.

54 f. : il. (color.) ; $30 \mathrm{~cm}$

Dissertação (mestrado)-Pontifícia Universidade Católica do Rio de Janeiro, Departamento de Administração, 2013.

Inclui bibliografia

1. Administração - Teses. 2. Opções de ações. 3. Volatilidade implícita. 4. Sorriso de volatilidade. 5. Superfície de volatilidade implícita. I. Pinto, Antonio Carlos Figueiredo II. Pontifícia Universidade Católica do Rio de Janeiro. Departamento de Administração. III. Título. 
A Marineia e Tatiana, mãe e irmã. 


\section{Agradecimentos}

A minha mãe e a minha irmã, Marineia e Tatiana Aguiar Fonseca, por tudo que precede à conclusão deste trabalho.

À memória de meu pai: Manoel Cordeiro Fonseca Filho e meus avós: Manoel, Haroldo e Valentina. A Vó Lourdes, meus familiares e amigos.

Ao meu orientador Prof. Antonio Carlos Figueiredo Pinto pela objetividade e tempestividade na passagem de conhecimento e apoio na orientação deste trabalho.

A Tatiana de Lima Cohe pela paciência, apoio e compreensão no transcorrer do curso até a conclusão deste trabalho. 


\section{Resumo}

Fonseca, Diego Aguiar; Pinto, Antonio Carlos Figueiredo. Previsibilidade na dinâmica da superfície de volatilidade implícita em opções de compra de ações brasileiras. Rio de Janeiro, 2013. 54p. Dissertação de Mestrado (Opção profissional) - Departamento de Administração, Pontifícia Universidade Católica do Rio de Janeiro.

O presente trabalho busca explorar a previsibilidade na dinâmica temporal em modelos lineares de superfícies de volatilidade implícita estimados para opções de compra de ações brasileiras. Resultados de estudos anteriores, sob a abordagem usualmente empregada de estimação de modelos lineares em função do preço de exercício e do tempo até o vencimento a partir de dados de corte transversal sobre cada contrato disponível em dado instante, como Dumas, Fleming e Whaley (1998), revelam grande instabilidade nos coeficientes estimados ao longo do tempo. Por conseguinte, a incapacidade desta perspectiva em descrever a dinâmica intertemporal da estrutura, contrariando a observação empírica de volatilidade variável no tempo. A partir destas evidências e das conclusões de Heston e Nandi (2000), que reportaram significativa dependência da trajetória para a volatilidade dos retornos do índice S\&P 500, Gonçalves e Guidolim (2006), propuseram um modelo em dois estágios, que aplica vetores autoregressivos para capturar a presença de variação temporal dos coeficientes de um modelo linear. A contribuição deste trabalho está em aplicar o Modelo proposto à realidade do mercado brasileiro de opções de ações, incipiente em liquidez e horizonte de negociação se comparado ao mercado norte americano, adaptando critérios a fim de validar sua aplicabilidade neste contexto em termos estatísticos e econômicos. Os resultados comprovam a superioridade desta abordagem em relação a outras comparáveis na literatura, mas não a capacidade de gerar retornos acima da média na presença de custos de transação contra a referência natural da taxa livre de risco. $\mathrm{O}$ que sugere a adequação à hipótese de eficiência de mercado.

\section{Palavras-chave}

Opções de ações; Volatilidade Implícita; Sorriso de Volatilidade; Superfície de Volatilidade Implícita. 


\section{Abstract}

Fonseca, Diego Aguiar; Pinto, Antonio Carlos Figueiredo (Advisor). Predicability dinamics in brazilian call options implied volatility surfaces. Rio de Janeiro, 2013. 54p. MSc. Dissertation - Departamento de Administração, Pontifícia Universidade Católica do Rio de Janeiro.

O The present study aims to explore predictability in temporal dynamics regarding linear models of the implied volatility surfaces estimated for Brazilian stocks options. Previous results, by usual approach of fitting linear models linking implied volatility to time to maturity and moneyness, available for each crosssection of option contracts at a point in time, as in Dumas, Fleming and Whaley (1998), suggest that estimated parameters of such models are highly unstable over time. Therefore, this approach isn't capable of replicating various IVS's shapes, contrary to the empirical evidence of implied volatility varying with options strike price and date of expiration. Based on these evidences and in Heston and Nandi (2000), that exploit the information on path-dependency in volatility contained in the spot S\&P 500 index, Gonçalves e Guidolim (2006) proposed a two-stage approach to modeling and forecasting the S\&P 500 index options IVS. In the second-stage they model the dynamics of the cross-sectional first-stage coefficients by means of vector autoregression models. The contribution of this work is to apply the proposed model to the reality of the Brazilian stock options, incipient in terms of liquidity and trading horizon dimensions when compared to the U.S. market, adapting criterians to validate its applicability in this context in statistical and economical sense. The results demonstrate the superiority of this approach over comparable literature, but not the ability to generate abnormal profits in the presence of transaction costs in excess of the benchmark of the riskfree rate. This indicates adaptation to the market efficiency hypothesis.

\section{Keywords}

Stock options; Implied Volatility; Volatility Smile; Implied Volatility Surface. 


\section{Sumário}

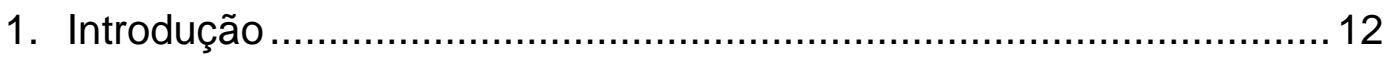

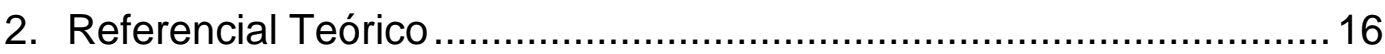

2.1. Modelo de Black e Scholes ………………............................. 16

2.2. Volatilidade Implícita, Sorriso de Volatilidade e Estrutura a

Termo de Volatilidade Implícita ............................................................. 18

2.3. Superfície de volatilidade Implícita .............................................22

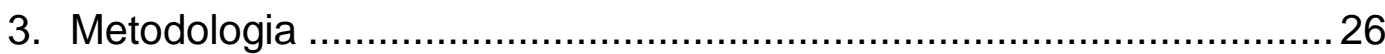

3.1. Dados e procedimentos de amostragem …………………........ 26

3.2. Estimativas de Volatilidade Implícita ………............................29

3.3. O Modelo para Superfície de volatilidade Implícita........................ 32

3.3.1. Resultados das Estimativas ............................................... 34

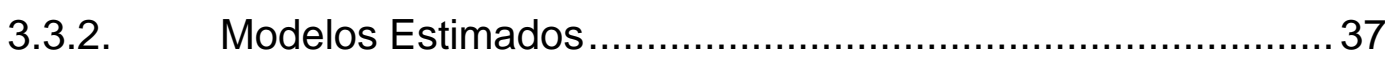

3.4. Medidas estatísticas e econômicas de análise ..............................39

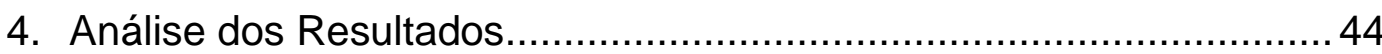

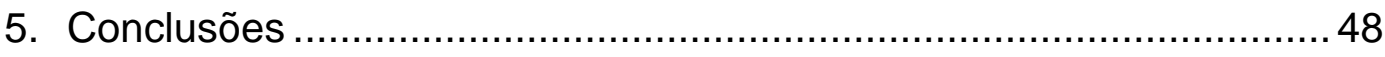

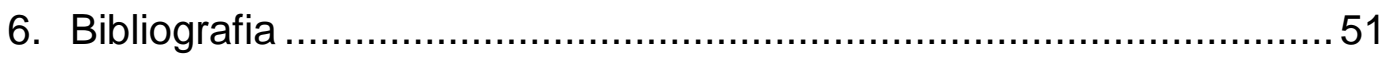




\section{Lista de tabelas}

Tabela 1 - Estatísticas descritivas por pregão na amostra .......................28

Tabela 2 - Vencimentos das opções negociadas por ativo ………….......29

Tabela 3 - Moneyness e vencimento das opções negociadas por ativo ..29

Tabela 4 - Volatilidade Implícita média por Moneyness e vencimento

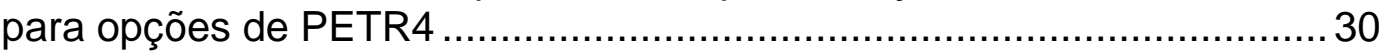

Tabela 5 - Volatilidade Implícita média por Moneyness e vencimento

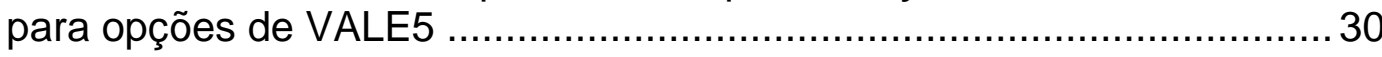

Tabela 6 - Volatilidade Implícita média por Moneyness e vencimento para opções de OGXP3

Tabela 7 - Volatilidade Implícita média por Moneyness e vencimento para opções de ITUB4

Tabela 8 - Estatísticas de coeficientes estimados em (1) para

PETR4

Tabela 9 - Estatísticas de coeficientes estimados em (1) para

VALE5

Tabela 10 - Estatísticas de coeficientes estimados em (1) para OGXP3.

Tabela 11 - Estatísticas de coeficientes estimados em (1) para

ITUB4

Tabela 12 - Resultados do teste ADF para estacionariedade das séries de coeficientes estimados.

Tabela 13 - Seleção de Modelo para VALE5 e PETR4 38

Tabela 14 - Janelas de estimativa e previsão para VALE5 e PETR4 40

Tabela 15 - Análise comparativa dos Modelos em dois estágios e DFW - contratos de VALE5 45

Tabela 16 - Análise comparativa dos Modelos em dois estágios e DFW - contratos de PETR4

Tabela 17 - RMSE por moneyness e vencimento para contratos sobre PETR4 
Tabela 18 - RMSE por moneyness e vencimento para contratos

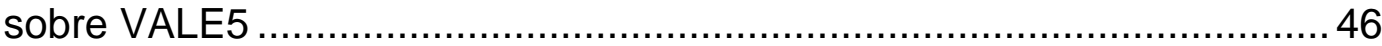

Tabela 19 - Resultados Econômicos das Estratégias .............................47 


\section{Lista de figuras}

Figura 1 - Correlação cruzada dos coeficientes estimados em (1) para PETR4

Figura 2 - Correlação cruzada dos coeficientes estimados em (1) para VALE5 35

Figura 3 - Superfícies Real e Estimada para PETR4

(21/07/2010 a 31/05/2012) e VALE5 (19/05/2010 a 15/03/2012) 39 